\title{
Joint Routing and Link Scheduling for Cognitive Radio Networks under Uncertain Spectrum Supply
}

\author{
Miao Pan*, Chi Zhang*, Pan $\mathrm{Li}^{\dagger}$ and Yuguang Fang* \\ ${ }^{*}$ Department of Electrical and Computer Engineering, University of Florida, Gainesville, FL 32611 \\ ${ }^{\dagger}$ Department of Electrical and Computer Engineering, Mississippi State University, Mississippi State, MS 39762
}

\begin{abstract}
The essential impediment to apply cognitive radio (CR) technology for spectrum utilization improvement lies in the uncertainty of licensed spectrum supply. In this paper, we investigate the joint routing and link scheduling problem of multihop CR networks under uncertain spectrum supply. We model the vacancy of licensed bands with a series of random variables, and introduce corresponding scheduling constraints and flow routing constraints for such a network. From a CR network planner/operator's point of view, we characterize the network with a pair of $(\alpha, \beta)$ parameters, and present a mathematical formulation with the goal of minimizing the required networkwide spectrum resource at the $(\alpha, \beta)$ level. Given that $(\alpha, \beta)$ is specified, we derive a lower bound for the optimization problem and develop a threshold based coarse-grained fixing algorithm for a feasible solution. Simulation results show that i) for any $(\alpha, \beta)$ level, the proposed algorithm provides a near-optimal solution to the formulated NP-hard problem; ii) the $(\alpha, \beta)$ based solution is better than expected bandwidth based one in terms of blocking ratio as well as spectrum utilization in CR networks.
\end{abstract}

\section{INTRODUCTION}

Booming growth of wireless networks and flourish of various wireless services have been witnessed in the past decade. In parallel with that, current static spectrum allocation policy of Federal Communications Commission (FCC) [1]-[3] results in the exhaustion of available spectrum, while a lot of licensed spectrum bands are extremely under-utilized. Experimental tests in academia [4], [5] and measurements conducted in industries [6], [7] both show that many licensed spectrum blocks are not used in certain geographical areas and are idle most of the time. Even in the most crowded area near downtown Washington, DC, where both government and commercial spectrum use is intensive, only $38 \%$ of the licensed spectrum remains occupied and the rest of spectrum resource (a.k.a., "white space/spectrum hole") is wasted. These statistics and studies spur the FCC to open up licensed spectrum bands and pursue new innovative technologies to encourage dynamic use of the under-utilized spectrum [1]. As one of the most promising solutions, cognitive radio (CR) technology releases the spectrum from shackles of authorized licenses, and enables the CR users to opportunistically access to the vacant licensed spectrum bands in either temporal or spatial domain.

Owing to the quick development of hardware designs, the advancement of radio technology and the maturity of signal processing [2], [8], [9], the frequency-agile CR devices are capable of exploring licensed spectrum bands, reconfiguring

This work was partially supported by the U.S. National Science Foundation under grants CNS-0916391 and CNS-0721744. The work of Y. Fang was also partially supported by the National Natural Science Foundation of China under grant 61003300 and the China 111 Project under Grant B08038.
$\mathrm{RF}$, switching frequencies across a wide spectrum range, sending and receiving packets over non-contiguous spectrum bands, etc. These desirable features of CR technology enable the CR users to opportunistically use the licensed spectrum when the primary services are not on, and greatly improve the utilization of spectrum resource. Consequently, CR technology promotes its numerous possible applications in various areas, e.g., military communications, public safety, disaster relief, search and rescue, environment monitoring and so on.

However, the key obstacle to the employment of multi-hop CR networks lies in the uncertain licensed spectrum supply [2], [3], [10]. Since the CR users must evacuate the licensed bands when primary services are active, the returning of primary services has significant impact on how to perform opportunistic spectrum accessing (OSA), scheduling and interference avoidance, and multi-hop multi-path routing in CR networks. State-of-the-art work on investigating the unpredictable activities of primary services can generally be classified into two categories: i) spectrum sensing and ii) statistical analysis of the collected/historical spectrum vacancy/occupancy data.

In spite of the overwhelming waste of sensing time which can be used for more traffic delivery, individual sensing is trapped by sensing accuracy since both false alarm probability and missing detection probability are really high. To overcome the weakness of individual sensing, cooperative sensing is proposed to improve the sensing accuracy by grouping CR users to sense together and share information among the group. But the trouble is that it is too difficult to synchronize the CR users in the group sensing simultaneously. In addition, cooperative sensing has to set up a common channel for information exchange, which will incur enormous communication overhead. On the other hand, in [4], [6], [7], researchers as well as engineers try to identify the spectrum supply for OSA with the statistics of licensed spectrum utilization rather than attempting to detect the activities of primary services. They have carried out spectrum measurements, collected and analyzed the data about spectrum utilization, and summarized the statistical characteristics of the band vacancy/occupancy in details. These statistical results contain abundant information about the activities of primary services and provide a nice guide to the CR users for OSA.

Resorting to the latter approach dealing with the unpredictable returning of primary services, in this paper, we focus on the joint routing and link scheduling problem for multihop CR networks under uncertain spectrum supply. Suppose there is a set of CR sessions characterized by a set of source destination pairs in the network, and each session has a certain 


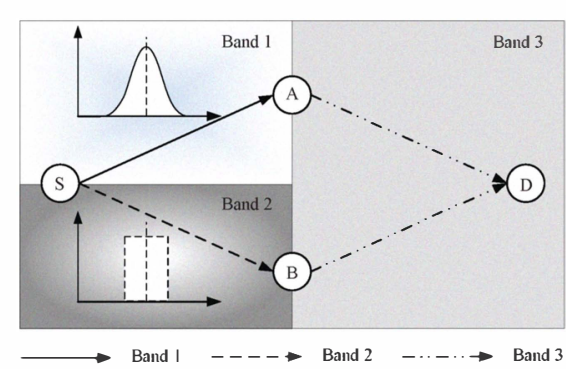

(a) Toy topology with one session.

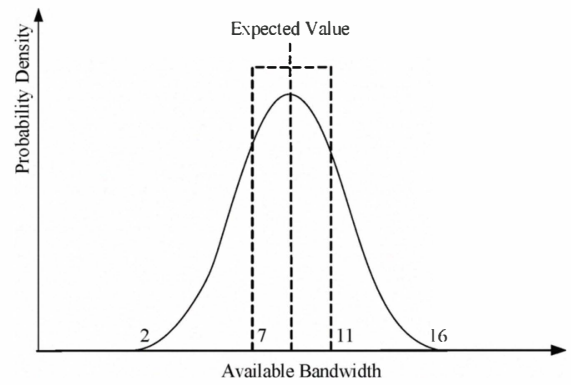

(b) The probability density functions of available bandwidth for band 1 and band 2 .

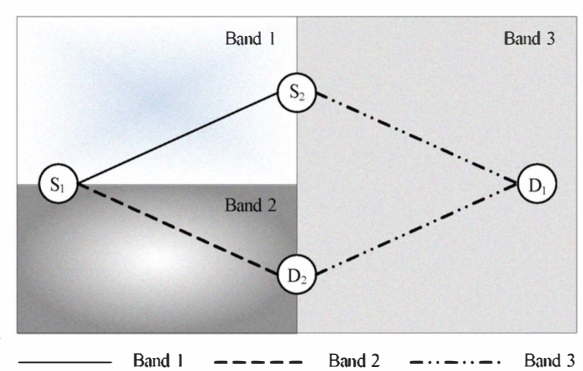

(c) Toy topology with two sessions.

Fig. 1. Simple examples for CR sessions under uncertain spectrum supply in CR networks.

rate requirement. Considering its geographical location, each CR node/user (in the sequel, we use the words node and user interchangeably) is able to opportunistically access to a set of licensed bands. The CR network planner/operator may ask an interesting question: how much bandwidth is at least required to maintain these CR sessions considering the availability of spectrum resources at a certain confidence level ${ }^{1}$ w.r.t. all the constraints from multiple layers in CR networks. To put it in another way, in this paper, we are trying to address how we can perform OSA, scheduling and multi-hop multi-path routing so that the required network-wide spectrum resource is minimized ${ }^{2}$, given the fact that the licensed spectrum supply cannot be guaranteed.

Inspired by the statistics of spectrum bands obtained on observation and experiments in [4] $]^{3},[6],[7]$, we novelly model the uncertain spectrum vacancy of a licensed band (i.e., available bandwidth for OSA) as a random variable satisfying certain distribution. This modeling explicitly distinguishes the joint routing and scheduling problem in CR networks from that in multi-channel multi-radio networks [14]-[17] or any other wireless networks [18], [19]. The reason is that in those networks the bandwidth is always regarded as a constant value. Even compared with prior work in the literature of multi-hop CR networks [11], [12], [20], the unique feature of uncertain spectrum supply makes the route selection and scheduling in our paper much more challenging as well. For example, suppose there is a toy CR network consisting of 4 CR nodes and 3 bands available for OSA. The source node can choose either the route $S-A-D$ or the route $S-B-D$ to deliver traffic as shown in Fig. 1(a). Moreover, assume the available bandwidth of Band 1 is normally distributed with $\mathcal{N}(9,6)$, the available bandwidth of Band 2 is uniformly distributed with $\mathcal{U}(7,11)$, and Band 3 is not the bottleneck for traffic delivery.

\footnotetext{
${ }^{1}$ The CR network planner/operator could be a general who intends to dispatch a squad of marines scattered at enemy territory using CR networks for communications, an engineer who wants to deploy a CR sensor network for environment monitoring, etc. Intuitively, the concern of such a planner is similar to the situation in the stock market, e.g., an investor often asks his broker how much a stock may increase by $10 \%$ at a certain confidence level.

${ }^{2}$ We follow the same objective as that in [11], [12], where the so-called space-bandwidth product defined in [13] is adopted as the performance metric in the setting of multi-hop CR networks.

${ }^{3}$ Chen et al. in [4] carried out a set of spectrum measurements in the $20 \mathrm{MHz}$ to $3 \mathrm{GHz}$ spectrum bands at 4 locations concurrently in Guangdong province of China. They used these data sets to conduct a set of detailed analysis about the statistics of the collected data, including channel occupancy/vacancy statistics, channel utilization, also spectral and spatial correlation of these measures.
}

An interesting question for the source CR node is which route is better. The answer is not straight-forward when the vacant bandwidth of Band 1 and that of Band 2 are represented by random variables. An intuitive solution is to evaluate the expected value of the available bandwidth, i.e., to measure which band can support larger flow rate or accommodate more flows on average ${ }^{4}$. In this case, consider the probability density functions (PDF) of bandwidth for the two bands, each random variable has an expected value of 9 as shown in Fig. 1(b), which makes the first order statistics based route selection implausible. Furthermore, consider the toy topology with two sessions in Fig. 1(c). Provided that all those licensed bands offer uncertain spectrum supply to CR nodes, we must identify how to calculate the sum of random variable for the scheduling and routing, which makes this problem in CR networks even more complex.

To formulate the problem mathematically, we exploit a pair of $(\alpha, \beta)$ parameters to characterize the network planner/operator's concerns about the CR network. Specifically, $\alpha$ denotes the targeted confidence level for the availability of the required network-wide spectrum resource, and $\beta$ denotes the targeted quality of CR communications. Besides, we demonstrate constraints from multiple layers under the situation that spectrum supply is uncertain. In particular, we pay special attention to modeling the unpredictable activities of primary services, scheduling and interference models, and multi-path routing constraints. We also dwell on how to integrate the bandwidth of different bands and calculate the sum of link capacity, when the vacant bandwidth of every licensed band is a random variable. We formulate an optimization problem with the objective of minimizing the required network-wide spectrum resource at an $(\alpha, \beta)$ level.

For a fixed pair of $(\alpha, \beta)$, the formulated optimization problem falls into a mixed integer non-linear programming and is proved to be NP-hard [21]. Aiming to derive a feasible solution, we present a sub-optimal algorithm for the NP-hard optimization. We first find a lower bound for the objective by relaxing the integer variables in scheduling and interference constraints. Then, we propose a coarse-grained fixing algorithm to iteratively determine binary integer variables exploiting a threshold, where the bandwidth integration and the sum of link capacity from different bands are computed using discrete Fourier transform (DFT) and inverse discrete

\footnotetext{
${ }^{4}$ The available bandwidth for OSA can directly be interpreted into link capacity using Shannon-Hartley theorem as illustrated in Sec. II-C2.
} 
Fourier transform (IDFT). As long as fixing all the integer variables, we can determine flow routing variables and solve the optimization problem. Since the solutions attained by the coarse-grained fixing algorithm is an upper bound for the optimization objective, we compare it with the lower bound we have developed earlier. Simulation results show that (i) the proposed coarse-grained fixing algorithm is near-optimal for any $(\alpha, \beta)$ level; (ii) compared with the expected bandwidth based solution, the $(\alpha, \beta)$ based one has better performance in the sense that it lowers down the blocking ratio of CR sessions and improves the spectrum utilization.

The rest of the paper is organized as follows. In Section II, we introduce the model of spectrum uncertainty and some other models in CR networks. In Section III, we mathematically describe scheduling and interference constraints and multi-hop multi-path routing in CR networks. In Section IV, We illustrate the bandwidth integration, define bandwidth required at $\alpha$, and formulate joint routing and scheduling as an NP-hard optimization problem. Besides, we find a lower bound for this optimization problem. In Section V, we develop a coarse-grained algorithm for a sub-optimal solution. Finally, we conduct simulations and analyze the performance results in Section VI, and draw concluding remarks in Section VII.

\section{NeTwORK MODEL}

\section{A. Opportunistic Spectrum Accessing}

We consider a multi-hop CR network consisting of $\mathcal{N}=$ $\{1,2, \cdots, n, \cdots, N\}$ CR nodes and a set of available licensed spectrum bands $\mathcal{M}=\{1,2, \cdots, m \cdots, M\}$ with unequal size of bandwidths ${ }^{5}$ as shown in Fig. 2. Suppose there are a set of $\mathcal{L}$ uni-cast communication sessions among these CR users. Let $s(l) / d(l)$ denote the source/destination node of session $l \in \mathcal{L}$, and $r(l)$ be the rate requirement of session $l$. The CR users are allowed to communicate with each other by opportunistically accessing to the licensed bands when the primary services are not active, but they must evacuate from these bands immediately when primary services become active.

Considering the geographical location of the CR nodes, the available spectrum bands at one node may be different from another node in the network. To put it in a mathematical way, let $\mathcal{M}_{i} \subseteq \mathcal{M}$ represent the set of available licensed bands at node $i \in \mathcal{N} . \mathcal{M}_{i}$ may be different from $\mathcal{M}_{j}$, where $j$ is not equal to $i$, and $j \in \mathcal{N}$, i.e., possibly $\mathcal{M}_{i} \neq \mathcal{M}_{j}$.

\section{B. Modeling of Uncertain Spectrum Supply}

The unique feature of CR networks is the uncertain spectrum supply from licensed bands, or say, the unpredictable bandwidth occupancy of primary services. To model this key feature of CR networks, we make $W^{m}$ denote the unoccupied bandwidth of licensed band $m \in \mathcal{M}$, where $W^{m}$ is a random variable considering the unpredictable activities of primary services. As shown in Fig. 2, generally speaking, people [22], [23] would like to use $\boldsymbol{E}\left(W^{m}\right)$, the first order statistics of $W^{m}$ [4] to predict the white space. Although this measurement

\footnotetext{
${ }^{5}$ Taking the least-utilized spectrum bands introduced in [12] [19] for example, we found that the bandwidth between $[1240,1300] \mathrm{MHz}$ (allocated to amateur radio) is $60 \mathrm{MHz}$, while bandwidth between [1525, 1710] $\mathrm{MHz}$ (allocated to mobile satellites, GPS systems, and meteorological applications) is $185 \mathrm{MHz}$.
}

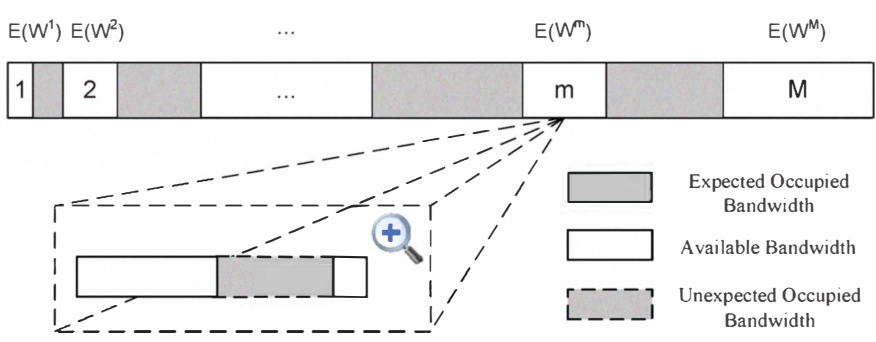

Fig. 2. A schematic illustrating available bandwidth for OSA and unpredictable occupation of primary services in CR networks.

is intuitive and easy to quantify, it ignores so much significant information w.r.t. the activities of primary services that it may lead to the failure of traffic delivery between CR nodes as depicted in the zoomed-in picture of Fig. 2. It should be noted that the statistical characteristics of $W^{m}$ contain abundant knowledge about the available bandwidth of band $m$ for CR users' opportunistic accessing. For example, assume $W^{m}$ is normally distributed with $\boldsymbol{E}\left(W^{m}\right)=2$ and $\sigma_{W^{m}}=1$, i.e., $W^{m} \sim \mathcal{N}\left(2,1^{2}\right)$. Then, the probability that $W^{m} \leq 3$ is equal to $84.1 \%$.

\section{Other Related Models}

1) Transmission Range and Interference Range: Suppose all CR nodes use the same power for transmission, and the power spectral density from the transmitter is $Q$. A widely used model [12], [20], [24] for power propagation gain is

$$
g_{i j}=\gamma \cdot d_{i j}^{-n}
$$

where $n$ is the path loss factor, $\gamma$ is an antenna related constant, and $d_{i j}$ is the distance between nodes $i$ and $j$. We assume that the data transmission is successful only if the received power spectral density at the receiver exceeds a threshold $Q_{T}$. Meanwhile, we assume interference becomes non-negligible only if it produces a power spectral density over a threshold of $Q_{I}$ at the receiver. Thus, the transmission range for a node is $R_{T}=\left(Q / Q_{T}\right)^{1 / n}$, which comes from $\left(R_{T}\right)^{-n} \cdot Q=Q_{T}$. Similarly, based on the interference threshold $Q_{I}\left(Q_{I}<Q_{T}\right)$, the interference range for a node is $R_{I}=\left(Q / Q_{I}\right)^{1 / n}$. It is obvious that $R_{I}>R_{T}$ since $Q_{I}<Q_{T}$.

2) Link Capacity: According to Shannon-Hartley theorem, if node $i$ sends data to node $j$ on link $(i, j)$ with band $m$, the capacity of link $(i, j)$ with band $m$ is

$$
c_{i j}^{m}=W^{m} \log _{2}\left(1+\frac{g_{i j} Q}{\eta}\right),
$$

where $\eta$ is the ambient Gaussian noise density. As we know, to mathematically model the link capacity is imperative in the sense that the aggregate flow rates on each radio link can never exceed this link's capacity, which is an important constraint for routing. Different from modeling of link capacity in the other wireless networks [19], [23] or in existing literature [12], [25], we are also aware that $c_{i j}^{m}$ is not a fixed number but a random variable since the available licensed bandwidth $W^{m}$ is uncertain in CR networks. Besides, note that the denominator inside the log function contains only $\eta$. This is because of one of our interference constraints, i.e., when node $i$ is transmitting to node $j$ on band $m$, then all the other neighbors of node $j$ within its interference range are prohibited from using this band. We will address the interference constraints in details in the following section. 


\section{SCheduling And Routing CONSTRAints FOR OPPORTUNISTIC ACCESSING}

\section{A. Link Scheduling and Interference Constraints}

Link scheduling can be conducted in time domain, in frequency domain, or in both of them. In this paper, we only focus on frequency based band assignment, i.e., how to assign bands at a CR node for transmission and reception. A plausible scheduling on frequency bands must consider the limitations at the transmitter side and guarantee no interference at the receiver side.

Assume band $m$ is available at both node $i$ and node $j$, i.e., $m \in \mathcal{M}_{i} \bigcap \mathcal{M}_{j}$. We denote

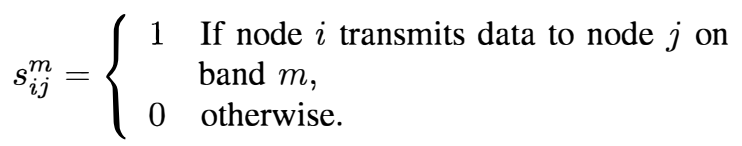

For a node $i \in \mathcal{N}$ and a band $m \in \mathcal{M}_{i}$, denote $\mathcal{T}_{i}^{m}$ the set of nodes that can also opportunistically access to band $m$ and are within the transmission range to node $i$, i.e.,

$$
\mathcal{T}_{i}^{m}=\left\{j: d_{i j} \leq R_{T}, j \neq i, m \in \mathcal{M}_{j}\right\} .
$$

From the view of the transmitter, node $i$ is not able to transmit to multiple nodes on the same frequency band. Thus, we have

$$
\sum_{q \in \mathcal{T}_{i}^{m}} s_{i q}^{m} \leq 1
$$

From the view of the receiver, a CR node cannot use the same frequency band for transmission and reception ${ }^{6}$, due to "self-interference" at the physical layer. That is, if $s_{i j}^{m}=1$, then for any $q \in \mathcal{T}_{j}^{m}, s_{j q}^{m}$ must be 0 , i.e.,

$$
s_{i j}^{m}+\sum_{q \in \mathcal{T}_{j}^{m}} s_{j q}^{m} \leq 1 .
$$

Note that in (6), we are referring to a specific node $j$ to which node $i$ is transmitting. If $s_{i j}^{m}=1$, then $\sum_{q \in \mathcal{T}_{j}^{m}} s_{j q}^{m}=0$, i.e., node $j$ is not able to use the same frequency band $m$ for transmission. On the other hand, if $s_{i j}^{m}=0$, then $\sum_{q \in \mathcal{T}_{j}^{m}} s_{j q}^{m} \leq 1$, i.e., node $j$ may use band $m$ for transmission, but can only use it for one receiving node $q \in \mathcal{T}_{j}^{m}$, which is the same as in (5).

Beyond the constraints above at the receiver, there are also interference constraints from the other nodes in the CR network. To be specific, for a frequency band $m$, if node $i$ uses this band for transmitting data to a node $j \in \mathcal{T}_{i}^{m}$, then any other node that may produce interference on node $j$ should not use this band ${ }^{7}$. To model this constraint, we let $\mathcal{P}_{j}^{m}$ represent the set of nodes that can produce interference at node $j$ on band $m$, i.e.,

$$
\mathcal{P}_{j}^{m}=\left\{p: d_{p j} \leq R_{I}, p \neq j, \mathcal{T}_{p}^{m} \neq \emptyset\right\} .
$$

The physical interpretation of $\mathcal{T}_{p}^{m} \neq \emptyset$ in the above formula is that node $p$ may use band $m$ for a valid transmission to a

\footnotetext{
${ }^{6}$ This limitation applies to both the transmitter and receiver. The reason to categorize it into the constraints of the receiver is for the ease of writing in the rest of paper. Also, as for this constraint, the roles of transmitter and receiver are symmetric and interchangeable.

7 "Hidden terminal" problem is a special case under this constraint.
}

node in $\mathcal{T}_{p}^{m}$ and then may cause interference to node $j$. Based on the definition of $\mathcal{P}_{j}^{m}$, we have

$$
s_{i j}^{m}+\sum_{q \in \mathcal{T}_{p}^{m}} s_{p q}^{m} \leq 1 \quad\left(p \in \mathcal{P}_{j}^{m}, p \neq i\right) .
$$

In (8), if $s_{i j}^{m}=1$, i.e., node $i$ uses band $m$ to transmit to node $j$, then any node $p$ that may interfere with node $j$ should not transmit on this band, i.e., $\sum_{q \in \mathcal{T}_{p}^{m}} s_{p q}^{m}=0$. Likewise, if $s_{i j}^{m}=0$, (8) reduces into (5), i.e., node $p$ may transmit on band $m$ to one node $q \in \mathcal{T}_{p}^{m}$, i.e., $\sum_{q \in \mathcal{T}_{p}^{m}} s_{p q}^{m} \leq 1$.

Now, we integrate the constraints in (6) and (8) into a general constraint at the receiver side. We define

$$
\mathcal{I}_{j}^{m}=\left\{p: d_{p j} \leq R_{I}, \mathcal{T}_{p}^{m} \neq \emptyset\right\},
$$

which is equivalent to

$$
\mathcal{I}_{j}^{m}= \begin{cases}\mathcal{P}_{j}^{m} \bigcup\{j\} & \text { If } \mathcal{T}_{j}^{m} \neq \emptyset \\ \mathcal{P}_{j}^{m} & \text { otherwise. }\end{cases}
$$

In this way, both (6) and (8) can be described by the following generalized constraint.

$$
s_{i j}^{m}+\sum_{q \in \mathcal{T}_{p}^{m}} s_{p q}^{m} \leq 1 \quad\left(p \in \mathcal{I}_{j}^{m}, p \neq i\right)
$$

\section{B. Routing Constraints}

As for routing, a source CR node may employ a number of relay nodes to forward the data packets toward its destination CR node. Obviously, there should be more than one path involved in data delivery since multi-path routing is more flexible to route the traffic from a source node to its destination. Following the routing model in [12], [25], we mathematically present the constraints at network layer as follows.

Let $f_{i j}(l)$ denote the data rate on link $(i, j)$ that is attributed to session $l$, where $i \in \mathcal{N}, j \in \bigcup_{m \in \mathcal{M}_{i}} \mathcal{T}_{i}^{m}$, and $l \in \mathcal{L}$. To simplify the notation, let $\mathcal{T}_{i}=\bigcup_{m \in \mathcal{M}_{i}} \mathcal{T}_{i}^{m}$.

If node $i$ is the source node of session $l$, i.e., $i=s(l)$, then

$$
\sum_{j \in \mathcal{T}_{i}} f_{i j}(l)=r(l)
$$

If node $i$ is an intermediate relay node for session $l$, i.e., $i \neq s(l)$ and $i \neq d(l)$, then

$$
\sum_{j \in \mathcal{T}_{i}}^{j \neq s(l)} f_{i j}(l)=\sum_{p \in \mathcal{T}_{i}}^{p \neq d(l)} f_{p i}(l) .
$$

If node $i$ is the destination node of session $l$, i.e., $i=d(l)$, then

$$
\sum_{p \in \mathcal{T}_{i}} f_{p i}(l)=r(l) .
$$

If (12) and (13) are satisfied, it can be easily verified that (14) must be satisfied. As a result, it is sufficient to list only (12) and (13) as routing constraints in the problem formulation.

In addition to the above flow balance equations at each node $i$ for each session $l$, the aggregate flow rates on each radio link cannot exceed this link's capacity, which is defined in (2). Taking interference constraints into consideration, the 
calculation of the link capacity $c_{i j}^{m}$ can be further simplified. When $s_{i j}^{m}=0$, we have $c_{i j}^{m}=0$. Thus, $c_{i j}^{m}$ should be written as

$$
c_{i j}^{m}=s_{i j}^{m} \cdot W^{m} \log _{2}\left(1+\frac{g_{i j} Q}{\eta}\right) .
$$

Therefore, for the requirement that the aggregate data rates on each link $(i, j)$ cannot exceed the link's capacity, we obtain

$$
\begin{aligned}
& \sum_{l \in \mathcal{L}}^{s(l) \neq j, d(l) \neq i} f_{i j}(l) \leq \sum_{m \in \mathcal{M}_{i} \cap \mathcal{M}_{j}} c_{i j}^{m} \\
= & \sum_{m \in \mathcal{M}_{i} \cap \mathcal{M}_{j}} s_{i j}^{m} \cdot W^{m} \log _{2}\left(1+\frac{g_{i j} Q}{\eta}\right) .
\end{aligned}
$$

\section{Problem Formulation ANd A Lower Bound For THE CROSS-LAYER OPTIMIZATION}

The essential objective of CR networks is to avoid the waste of "white space" and to improve the spectrum utilization. To put it in another word, for the given amount of radio resource, we try to use it to support as many CR users' sessions as possible; correspondingly, for the given number of CR users' sessions, we try to use as little radio resource as possible to support them. In this paper, we measure the radio resource in terms of the total bandwidth required by the network planner/operator to support a set of CR sessions, which is the simplified form of the so called space-bandwidth product proposed in [13] with fixed transmission power.

As introduced in Sec. II, there is a set of source and destination pairs (CR users' sessions) in the network, each with a certain rate requirement. Each node is entitled to opportunistically access to a set of licensed spectrum bands with uncertain supply for communications. We seek for a feasible solution to assigning the available frequency bands to each node, scheduling bands for transmission and reception, and routing the flows so that the total radio bandwidth required in the multi-hop CR network is minimized.

Intuitively, the optimization problem can be formulated as follows [11], [12].

$$
\begin{gathered}
\text { Min } \sum_{i \in \mathcal{N}} \sum_{m \in \mathcal{M}_{i}} \sum_{j \in \mathcal{T}_{i}^{m}} W^{m} s_{i j}^{m} \\
\text { s.t. } \quad \sum_{q \in \mathcal{T}_{i}^{m}} s_{i q}^{m} \leq 1 \quad\left(i \in \mathcal{N}, m \in \mathcal{M}_{i}\right) \\
s_{i j}^{m}+\sum_{\substack{q \in \mathcal{T}_{p}^{m} \\
s(l)}} s_{p q}^{m} \leq 1\left(i \in \mathcal{N}, m \in \mathcal{M}_{i}, j \in \mathcal{T}_{i}^{m}, p \in \mathcal{I}_{j}^{m}, p \neq i\right) \\
\sum_{l \in \mathcal{L}}^{s(l) \neq i} f_{i j}(l)-\sum_{m \in \mathcal{M}_{i} \cap \mathcal{M}_{j}} W^{m} \log _{2}\left(1+\frac{g_{i j} Q}{\eta}\right) s_{i j}^{m} \leq 0 \\
\sum_{j \in \mathcal{T}_{i}} f_{i j}(l)=r(l) \\
\sum_{j \in s, \mathcal{T}_{i}} f_{i j}(l)-\sum_{p \in \mathcal{T}_{i}} f_{p i}(l)=0 \quad\left(l \in \mathcal{N}, j \in \mathcal{T}_{i}\right) \\
s_{i j}^{m}=0 \text { or } 1, f_{i j}(l) \geq 0(l \in \mathcal{L}, i=s(l)) \\
\end{gathered}
$$

where $s_{i j}^{m}$ and $f_{i j}(l)$ are optimization variables, and $g_{i j}, Q, \eta$ and $r(l)$ are all constants.

Note that due to the unpredictable returning of primary services, $W^{m}$ is not modeled as a constant but modeled as a random variable in CR networks as illustrated in Sec. II-C. This feature makes the spectrum resource minimization problem in this paper far different from that with guaranteed spectrum supply in existing works [12], [25]. Therefore, two critical issues need to be addressed in the intuitive formulation above.

First, bandwidth integration in (17) and (18) is the sum of a series of random variables in CR networks rather than the sum of a series of deterministic quantities in other kinds of wireless networks.

Second, with different choices of $s_{i j}^{m}$ and $f_{i j}(l)$, we have difficulty in comparing results of the optimization, i.e., $\sum_{i \in \mathcal{N}} \sum_{m \in \mathcal{M}_{i}} \sum_{j \in \mathcal{T}_{i}^{m}} W^{m} s_{i j}^{m}$, because they are random variables with different kinds of distribution.

\section{A. Problem Formulation}

1) Bandwidth Integration: We take a simple example to illustrate how to integrate the bandwidth of different bands. We let $W^{c}=W^{a}+W^{b}$, where $W^{a}$ and $W^{b}$ are independent ${ }^{8}$, and bands $a, b \in \mathcal{M}$. Furthermore, we assume the probability density function (PDF) and cumulative distribution function (CDF) of $W^{a}$ and $W^{b}$ are $h_{W^{a}}\left(w^{a}\right), h_{W^{b}}\left(w^{b}\right), H_{W^{a}}\left(w^{a}\right)$ and $H_{W^{b}}\left(w^{b}\right)$, respectively. Then, the CDF and PDF of $W^{c}$ are derived as follows.

$$
\begin{aligned}
H_{W^{c}}\left(w^{c}\right) & =\operatorname{Pr}\left(W^{c} \leq w^{c}\right) \\
& =\operatorname{Pr}\left(W^{a}+W^{b} \leq w^{c}\right) \\
& =\int_{-\infty}^{\infty} \int_{-\infty}^{w^{c}-w^{a}} h_{W^{a}, W^{b}}\left(w^{a}, w^{b}\right) d w^{b} d w^{a} .
\end{aligned}
$$

Given $W^{a}$ and $W^{b}$ are independent, we further calculate

$$
\begin{aligned}
H_{W^{c}}\left(w^{c}\right) & =\int_{-\infty}^{\infty} \int_{-\infty}^{w^{c}-w^{a}} h_{W^{a}}\left(w^{a}\right) h_{W^{b}}\left(w^{b}\right) d w^{b} d w^{a} \\
& =\int_{-\infty}^{\infty} h_{W^{a}}\left(w^{a}\right) H_{W^{b}}\left(w^{c}-w^{a}\right) d w^{a} .
\end{aligned}
$$

Moreover, the probability density of $W^{c}$ is expressed as

$$
\begin{aligned}
h_{W^{c}}\left(w^{c}\right) & =\int_{-\infty}^{\infty} h_{W^{a}}\left(w^{a}\right) \frac{\partial H_{W^{b}}\left(w^{c}-w^{a}\right)}{\partial w^{c}} d w^{a} \\
& =\int_{-\infty}^{\infty} h_{W^{a}}\left(w^{a}\right) h_{W^{b}}\left(w^{c}-w^{a}\right) d w^{a} .
\end{aligned}
$$

Thus, $h_{W^{c}}\left(w^{c}\right)$ is the convolution of $h_{W^{a}}\left(w^{a}\right)$ and $h_{W^{b}}\left(w^{b}\right)$ [26]. It can be written as

$$
h_{W^{c}}\left(w^{c}\right)=h_{W^{a}}\left(w^{a}\right) * h_{W^{b}}\left(w^{b}\right)=\bigotimes_{m \in\{a, b\}} h_{W^{m}}\left(w^{m}\right),
$$

where $\otimes$ denotes the operator for the convolution of a sequence. From the calculation of $h_{W^{c}}\left(w^{c}\right)$, we find that the sum of two independent random variables is associative and commutative. Using the same approach as in (19), (20) and (21), this property can easily be extended to the sum

\footnotetext{
${ }^{8}$ This assumption is held for any two bands in CR networks for the whole paper.
} 
of a finite number of random variables. For example, for the bandwidth integration of link $(i, j)$, the PDF of $W=$ $\begin{aligned} & \sum_{m \in \mathcal{M}_{i} \cap \mathcal{M}_{j}} W^{m} s_{i j}^{m} \text { is } \\ & h_{W}(w)=\bigotimes_{m \in \mathcal{M}_{i} \cap \mathcal{M}_{j}} h_{W^{m}}\left(w^{m}\right) s_{i j}^{m} .\end{aligned}$

2) Bandwidth Required at $\alpha$ : Before we re-formulate the problem, we must quantify the bandwidth required for OSA when the vacancy of the licensed band is uncertain and modeled as a random variable. Thus, we leverage parameter $\alpha$ to define bandwidth required at $\alpha$ for OSA. Inspired by the mathematical expression of value at risk ( VaR) in [27], we use $X_{\alpha}(w)$ to denote bandwidth required at $\alpha$ and define it as follows.

$$
\left\{\begin{array}{lll}
H_{W}(\tau)=\int_{-\infty}^{\tau} h_{W}(w) d w, & & \tau \in \mathcal{R} \\
X_{\alpha}(W)=\inf \left\{\tau: H_{W}(\tau) \geq \alpha\right\}, & & \alpha \in[0,1] .
\end{array}\right.
$$

From (24), we find that the available bandwidth of the licensed bandwidth integration for OSA is less than $X_{\alpha}(W)$ at confidence level $\alpha$ as shown in Fig. 3(a).

3) Formal Formulation: Based on the description of bandwidth integration and definition of bandwidth required at $\alpha$, the optimization problem can be reformulated as follows.

$$
\begin{aligned}
& \text { Min } X_{\alpha}\left(\sum_{i \in \mathcal{N}} \sum_{m \in \mathcal{M}_{i}} \sum_{j \in \mathcal{T}_{i}^{m}} W^{m} s_{i j}^{m}\right) \\
& \text { s.t. } \quad \sum_{q \in \mathcal{T}_{i}^{m}} s_{i q}^{m} \leq 1 \quad\left(i \in \mathcal{N}, m \in \mathcal{M}_{i}\right) \\
& s_{i j}^{m}+\sum_{q \in \mathcal{T}_{p}^{m}} s_{p q}^{m} \leq 1 \quad\left(i \in \mathcal{N}, m \in \mathcal{M}_{i}, j \in \mathcal{T}_{i}^{m},\right. \\
& \left.p \in \mathcal{I}_{j}^{m}, p \neq i\right) \\
& \operatorname{Pr}\left(\sum_{l \in \mathcal{L}}^{s(l) \neq j, d(l) \neq i} f_{i j}(l) \leq \sum_{m \in \mathcal{M}_{i} \cap \mathcal{M}_{j}} W^{m} \log _{2}\left(1+\frac{g_{i j} Q}{\eta}\right) s_{i j}^{m}\right) \geq \beta \\
& \left(i \in \mathcal{N}, j \in \mathcal{T}_{i}\right) \\
& \sum_{j \in \mathcal{T}_{i}} f_{i j}(l)=r(l) \\
& (l \in \mathcal{L}, i=s(l)) \\
& \sum_{j \in \mathcal{T}_{i}}^{j \neq s(l)} f_{i j}(l)-\sum_{p \in \mathcal{T}_{i}}^{p \neq d(l)} f_{p i}(l)=0 \quad(l \in \mathcal{L}, i \in \mathcal{N}, i \neq s(l), d(l)) \\
& s_{i j}^{m}=0 \text { or } 1, f_{i j}(l) \geq 0\left(l \in \mathcal{L}, i \in \mathcal{N}, i \neq d(l), j \in \mathcal{T}_{i}, j \neq s(l)\right) .
\end{aligned}
$$

Compared with the intuitive formulation, the reformulated problem mathematically solves the sum of random variables by using bandwidth integration and incorporates the other parameter $\beta$ to represent the network planner/operator's requirements about quality of CR communications as presented in (27).

In addition, the objective of the optimization is clarified, i.e., to minimize bandwidth required at $\alpha$ to support the CR sessions with rate requirements, when joint scheduling and routing constraints are satisfied. Take $W$ and $W^{\prime}$ in Fig. 3(b) for example, assume they are both integrated bandwidths which satisfy all the constraints listed above. We can choose either $W$ or $W^{\prime}$ for OSA. We compare $X_{0.9}(W)$ and $X_{0.9}\left(W^{\prime}\right)$ as shown in Fig. 3(b), and decide to use $W$ for OSA. The reason is that if we choose $W$, the available bandwidth of licensed
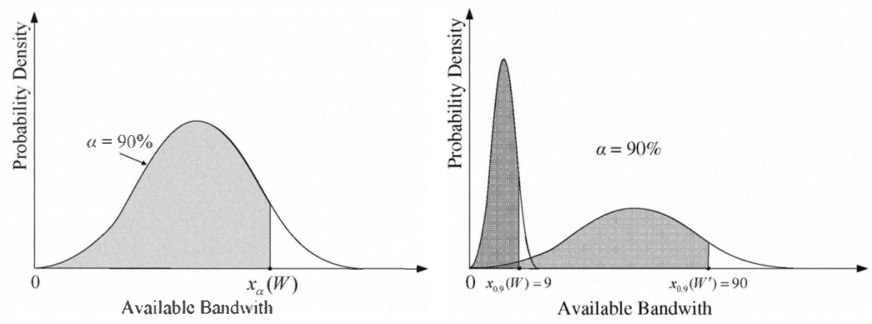

(a) The geometrical illustration of (b) The geometrical illustration of op$X_{\alpha}(w)$. timization objective.

Fig. 3. Bandwidth required at $\alpha$ and optimization objective.

bandwidth integration is less than $X_{0.9}(W)=9$ at confidence level of $90 \%$; but if we choose $W^{\prime}$, the available bandwidth of licensed bandwidth integration is less than $X_{0.9}\left(W^{\prime}\right)=90$ at confidence level of $90 \%$. At an $(\alpha, \beta)$ level, the smaller $X_{\alpha}(W)$ is, the less spectrum required to maintain the set of $\mathrm{CR}$ sessions. The less spectrum required, the less CR sessions are affected by the activities of primary services.

Nevertheless, the above optimization problem itself is a mixed-integer nonlinear programming problem, which is proved to be NP-hard [21].

\section{B. The Lower Bound for the Cross-layer Optimization}

For an arbitrary pair of $(\alpha, \beta)$, the complexity of the problem formulated in Sec. IV-A arises from the binary $s_{i j}^{m}$ variables. To reduce the complexity and pursue a lower bound for the cross-layer optimization, we relax the binary requirement on $s_{i j}^{m}$ and replace it with $0 \leq s_{i j}^{m} \leq 1$. Due to the enlarged optimization space (caused by relaxation on $s_{i j}^{m}$ ), the solution to this relaxed optimization problem yields a lower bound for the minimization of bandwidth required at $\alpha$ problem in Sec. IV-A. Although the lower bound may not be achieved by a feasible solution, it offers a benchmark to measure the quality of feasible solutions.

\section{A FASt Fixing Algorithm FOR SUb-Optimal SOLUTIONS USING DFT-IDFT}

Whereas we have the lower bound as the benchmark, we still seek for an effective and efficient solution to the proposed problem since the $s_{i j}^{m}$ variables are binary values rather than real numbers within 0 and 1 . Given the values of the $(\alpha, \beta)$ pair, in this section, we first investigate how to reduce the complexity of computing the PDF convolution involved in the bandwidth integration. Then, with the knowledge of bandwidth integration computation, we present a coarse-grained fixing procedure to produce a feasible solution to the cross-layer optimization problem [11], [12].

\section{A. Fast Computation of the Bandwidth Integration}

Following the typical way to efficiently calculate the linear convolution in [28], we implement the PDF convolution of bandwidth integration in Sec. IV-A in four steps.

Briefly speaking, we firstly convert the continuous PDF of $W^{m}$ into a discrete sequence by periodic sampling. Then, we zero-pad all the sequences, and compute the DFT of each sequence using the fast Fourier transform (FFT) algorithm (e.g., Cooley-Tukey algorithm). After that, we point-by-point multiply the DFTs of all the sequences, where the product 
represents the DFT of the PDF convolution of $W^{m}$. Finally, we compute the IDFT of the product, and convert the discrete result into continuous one to reconstruct the PDF convolution of the bandwidth integration.

\section{B. The Coarse-grained Fixing Procedure}

Now, the left problem is how to determine the $s_{i j}^{m}$ variables and fix flow routing in the problem formulation in Sec. IV-A. The key to simplifying the NP-hard optimization, fixing $f_{i j}(l)$ variables, and attaining an effective solution is the determination of the binary values for the $s_{i j}^{m}$ variables [11], [12].

To determine the values of all the $s_{i j}^{m}$-variables, we iteratively solve a sequence of relaxed optimization problems. Considering interference constraints, in each iteration, we can fix at least one binary value for some $s_{i j}^{m}$. Specifically, for the first iteration, we relax all binary variables $s_{i j}^{m}$ to $0 \leq s_{i j}^{m} \leq 1$ as in Sec. IV-B to obtain a new optimization problem. We integrate the bandwidth with the PDF convolution and solve this new problem, so that we have a solution with each $s_{i j}^{m}$ being a value between 0 and 1 . Then, we select the $s_{i j}^{m}$ with the largest value among all the $s_{i j}^{m}$-values, and set this particular $s_{i j}^{m}$ to be 1 . In parallel with this fixing, by (25), we should set $s_{i q}^{m}=0$ for $\left(q \in \mathcal{T}_{i}^{m}, q \neq j\right)$. Meanwhile, by (26), we should set $s_{p q}^{m}$ to 0 for $\left(p \in \mathcal{I}_{j}^{m}, p \neq i, q \in \mathcal{T}_{p}^{m}\right)$. In particular, if the result includes more than one $s_{i j}^{m}$-variables with the value of 1 , we can set those $s_{i j}^{m}$-variables to 1 and perform an additional fixing for the largest fractional variable in the current iteration as illustrated above.

Having fixed some $s_{i j}^{m}$-variables in the first iteration, we remove all the terms associated with those already fixed $s_{i j}^{m}$ variables, eliminate the related constraints in (25) and (26), and update the problem to a new one for the second iteration. In the second iteration, we solve the new optimization and then determine the values of some other unfixed $s_{i j}^{m}$-variables based on the same process ${ }^{9}$. The iteration continues until we fix all $s_{i j}^{m}$-variables to be either 0 and 1 . The overall grained fixing procedure is summarized in Alg. 1.

Considering the number of bands with different frequencies and the spatial reuse in multi-hop CR networks, we may further reduce the complexity of the algorithm and speed up the procedure by fixing more $s_{i j}^{m}$-variables in a coarse-grained manner during each iteration. Firstly, the transmission in one band has no interference impact on the transmission in any other bands with different frequencies. Thus, for a link $(i, j)$, we may fix multiple bands within a single iteration in the coarse-grained fixing algorithm. Then, from the view of spatial reuse, a band can be used by the links far apart from one another (i.e., beyond the interference range of the nodes in communications with a link). Thus, for a band $m$, we may fix multiple links that have no mutual interference within a single iteration in the coarse-grained fixing algorithm.

To be specific, we employ a threshold $\theta>0.5$ in the coarsegrained fixing process and fix all the $s_{i j}^{m}$-variables exceeding $\theta$ to 1 in a single iteration. To make sure that the constraints in (25) and (26) are held in the relaxed problem, we find that

\footnotetext{
${ }^{9}$ Provided that some $s_{i j}^{m}$-variables are fixed in the first iteration, the computation complexity in the second iteration is lower than that in the first iteration because we only need to deal with the remaining un-fixed $s_{i j}^{m}$ variables
}

at most one variable $s_{i j}^{m}$ is allowed to be larger than $\theta$ in the local area in CR networks. Therefore, $\theta>0.5$ is suitable for determining the binary values of $s_{i j}^{m}$-values. In the case that none of the $s_{i j}^{m}$-variables exceed $\theta$, we will resort to the procedure listed in Alg. 1 for the current iteration and set the largest valued $s_{i j}^{m}$-variable to 1 .

Different from the lower bound obtained in Sec. IV-B, the proposed fast algorithm yields an upper bound to the problem formulated in Sec. IV-A. The quality of our suboptimal approach can be assessed by comparing its solution to the lower bound at various $(\alpha, \beta)$ levels.

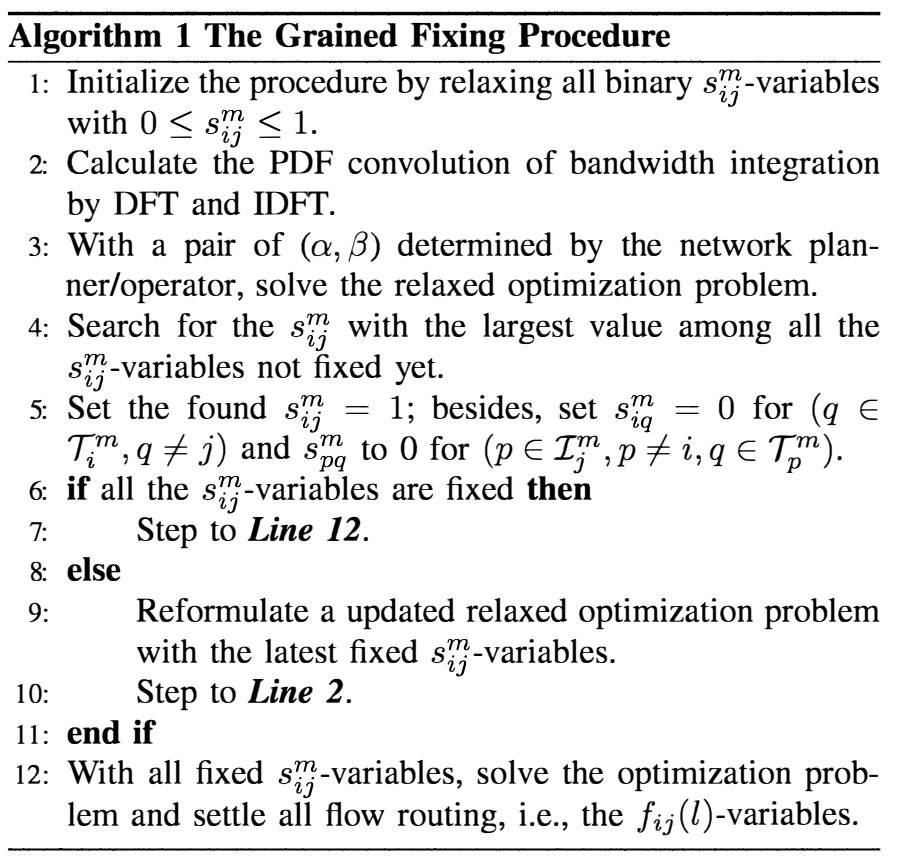

\section{PERformance AnAlysis}

\section{A. Simulation Setup}

We conduct simulations with a $\mathrm{CR}$ network consisting of $|\mathcal{N}|=25 \mathrm{CR}$ nodes in a $50 * 50 \mathrm{~m}^{2}$ area. Among these nodes, there are $|\mathcal{L}|=6$ active $\mathrm{CR}$ sessions, each session with a random rate requirement within $[10,100] \mathrm{Mb} / \mathrm{s}$. We assume that the transmission range of each node is $20 \mathrm{~m}$, that the interference range is $30 \mathrm{~m}$, and that the path loss index $n$ is 4 . For the simplicity of computation [11], [12], we assume the threshold $Q_{T}$ is equal to the ambient Gaussian noise density, i.e., $\eta$. Thus, we have $Q_{I}=\left(\frac{20}{30}\right)^{n} Q_{T}$ and the transmission power spectral density $Q=(20)^{n} Q_{T}=1.6 \cdot 10^{5}$ according to the analysis in Sec. II-C.

As for the uncertain spectrum supply, we assume that there are $|\mathcal{M}|=20$ licensed bands that can be opportunistically used by $\mathrm{CR}$ nodes in the whole network. The vacant bandwidths of these bands are represented by a series of random variables. Based on data collected and the statistical analysis on spectrum utilization in [4], those random variables are exponentially distributed ${ }^{10}$, i.e., $h_{W^{m}}\left(w^{m}, \lambda^{m}\right)=\lambda^{m} e^{-\lambda^{m} w^{m}}$, where $\lambda^{m} \in$ $(0,3]$. As we know, available bands for each CR node are a

\footnotetext{
${ }^{10}$ The results and analysis can easily be extended to other distributions (e.g., normal distribution, uniform distribution, etc.), even for the case that $W^{m}$ from different spectrum bands satisfies different distributions.
} 


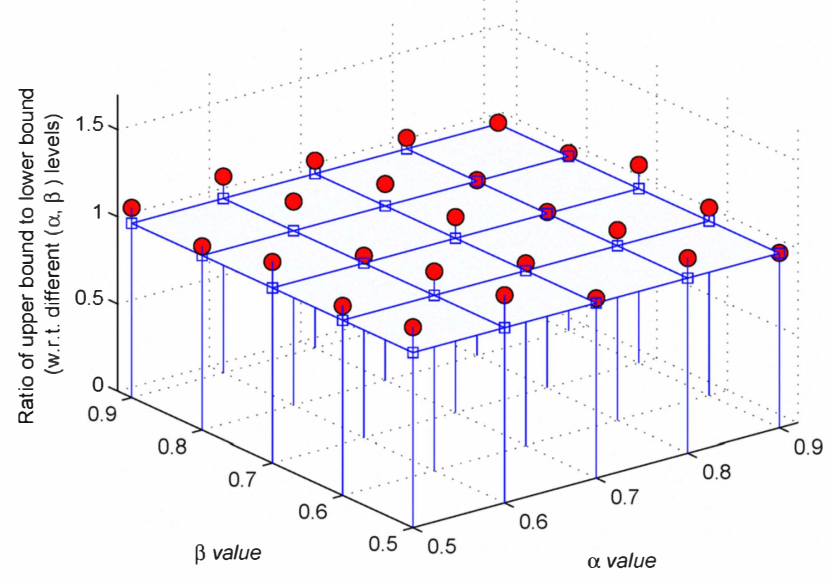

Fig. 4. Ratio of the upper bound to the lower bound at various $(\alpha, \beta)$ levels.

subset of these 20 bands based on its location, and the available bands for any two CR nodes in the network may not be the same. Therefore, we randomly select a subset of bands from the spectrum pool of 20 bands for each node in the simulations. Due to different $\lambda^{m}$-values and random selection process, the size of available bandwidth in each band may be unequal, which truthfully mirrors the practical scenario.

It is not surprising that there may exist no feasible solution for some specific data set, because of dis-connectivity, inherent resource bottleneck in a hot spot, etc. In this paper, we only focus on the data sets with feasible solutions and analyze the corresponding results as shown in the next subsection.

\section{B. Results and Analysis}

In Fig. 4, we evaluate the proposed coarse-grained fixing algorithm. We set $\theta=0.75$ (i.e., the threshold for the coarsegrained fixing), and compare the upper bound determined by the coarse-grained fixing algorithm with the lower bound developed in Sec. IV-B at different $(\alpha, \beta)$ levels. The range of $(\alpha, \beta)$ values is from $(50 \%, 50 \%)$ to $(90 \%, 90 \%)$, and simulations for the comparison of bounds are conducted for every $10 \%$ increase in either $\alpha$ or $\beta$ value. For each pair of $(\alpha, \beta)$, we employ 50 data sets that can produce feasible solutions and take the average value as a result. For each data set, we re-generate the network topology, source/destination pair and bit rate of each session, and available frequency bands for each CR node, which follows the guideline of simulation setup. As shown in Fig. 4 (the ratio is denoted by balls in shade; the benchmark of value 1 is denoted by the contour area intercepted and by the hollow squares at the sampled $(\alpha, \beta)$ pairs), the ratio of the upper bound to the lower bound via integer relaxation is equal to or slightly above to 1 in almost all the area. The average ratio of the upper bound to the lower bound for all the sampled data sets is 1.0506, and the standard deviation is 0.0867 . It indicates that since the ratio of the upper bound to the lower bound is close to 1 at any $(\alpha, \beta)$ level, and the optimal bandwidth required at $\alpha$ is between those bounds, the solution found by the coarse-grained fixing algorithm must be close to the optimum.

Figure 5 shows the comparison between the proposed $(\alpha, \beta)$ based approach and the expected bandwidth based approach in

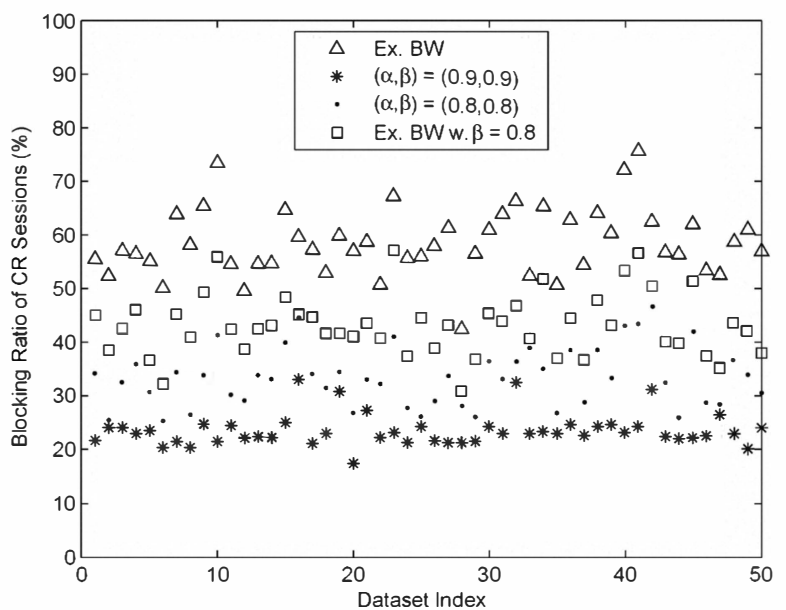

Fig. 5. The blocking ratio of different approaches.

which the expected value of bandwidth is used to characterize both the objective of the optimization and corresponding constraints [22]. For illustrative purposes, we compare the solution of the expected bandwidth based approach with the solutions obtained by the coarse-grained fixing algorithm at $(\alpha, \beta)=(80 \%, 80 \%),(\alpha, \beta)=(90 \%, 90 \%)$ and $\beta=80 \%$ with the expected value of required bandwidth as the objective, respectively. We take the blocking ratio of CR sessions as the evaluation metric and present simulation results for 50 data sets. From the results shown in Fig. 5, three observations can be made in order. First, the performance of the expected bandwidth based approach is worst of all because it ignores both the uncertainty of spectrum supply and the quality of CR communications when it selects the subset of licensed bands satisfying scheduling and routing constraints for OSA. The expected bandwidth with $\beta=80 \%$ approach is worse than the $(\alpha, \beta)$ based one because it also neglects the uncertain spectrum supply, i.e., the availability of required spectrum, as illustrated in Sec. IV-A3. By contrast, taking both factors into consideration, the $(\alpha, \beta)$ based approach performs the best. Second, for the $(\alpha, \beta)$ based approach, the blocking ratio decreases as the $(\alpha, \beta)$ level increases. Third, since the blocking ratio of $\mathrm{CR}$ sessions is closely associated with the spectrum utilization ratio in CR networks, i.e., low blocking ratio is equivalent to high spectrum utilization ratio for a given set of $\mathrm{CR}$ sessions. We can claim that the $(\alpha, \beta)$ based approach is better than the expected bandwidth based one in terms of spectrum utilization as well.

With a specific set of CR sessions (i.e., the network topology, the source/destination pair and the rate requirement of each session are fixed), Table I presents a general trend of change in terms of the bandwidth required at $\alpha$ in $\mathrm{CR}$ networks at different $(\alpha, \beta)$ levels. It is obvious that as $\alpha$ value increases, both the lower bound and the upper bound of the bandwidth required at $\alpha$ increase. Similarly, as $\beta$-value increases, both the bounds of the bandwidth required at $\alpha$ increase as well. The reason is from two aspects: i) From the optimization objective's point of view, the larger $\alpha$, the higher confidence level the network operator requests for the availability of required spectrum. The higher confidence level, 
TABLE I

LOWER AND UPPER BOUNDS OF THE BANDWIDTH REQUIRED AT $\alpha$ FOR A GIVEN SET OF CR SESSIONS AT DIFFERENT $(\alpha, \beta)$ LEVELS.

\begin{tabular}{|c|c|c|c|c|}
\hline Index & $\alpha(\%)$ & $\beta(\%)$ & Lower-Bs & Upper-Bs \\
\hline 1 & 70 & 80 & 481.88 & 496.09 \\
\hline 2 & 75 & 80 & 517.29 & 517.29 \\
\hline 3 & 80 & 80 & 566.39 & 594.37 \\
\hline 4 & 85 & 80 & 628.71 & 631.15 \\
\hline 5 & 90 & 80 & 669.76 & 690.05 \\
\hline 6 & 70 & 85 & 490.28 & 493.25 \\
\hline 7 & 75 & 85 & 562.89 & 573.30 \\
\hline 8 & 80 & 85 & 589.02 & 595.13 \\
\hline 9 & 85 & 85 & 662.54 & 664.74 \\
\hline 10 & 90 & 85 & 714.15 & 725.87 \\
\hline 11 & 70 & 90 & 503.32 & 506.09 \\
\hline 12 & 75 & 90 & 602.34 & 606.50 \\
\hline 13 & 80 & 90 & 613.90 & 631.76 \\
\hline 14 & 85 & 90 & 681.88 & 696.09 \\
\hline 15 & 90 & 90 & 735.07 & 741.68 \\
\hline
\end{tabular}

the more bandwidth required at $\alpha$ is needed. ii) From the constraint's point of view, the larger $\beta$, the better quality of communications in $\mathrm{CR}$ networks. The better quality of $\mathrm{CR}$ communications, the more bandwidth required at $\alpha$ is needed, provided that the set of CR sessions is identified.

\section{CONCLUSION}

In this paper, we have presented a theoretical study on the joint frequency scheduling and routing problem in multi-hop CR networks under uncertain spectrum supply. Enlightened by the statistics of spectrum utilization, we first model the vacancy of a licensed band with a random variable satisfying certain statistical distribution. Then, we elaborate on scheduling and interference constraints as well as routing constraints w.r.t. the unpredictable activities of primary services. After that, we characterize the network with a pair of $(\alpha, \beta)$ parameters, and present a mathematical formulation with the goal of minimizing the required network-wide spectrum resource at a $(\alpha, \beta)$ level for a set of $\mathrm{CR}$ sessions with rate requirements. Since the formulated optimization problem is NP-hard, we derive a lower bound for the objective by relaxing the integer variables. Furthermore, we propose a coarse-grained fixing algorithm for a feasible solution. Through simulations, we show that the solution attained by the proposed algorithm is near-optimal to the formulated NP-hard problem at any $(\alpha, \beta)$ level; meanwhile, the $(\alpha, \beta)$ based solution is better than expected bandwidth based one in terms of blocking ratio and spectrum utilization.

\section{REFERENCES}

[1] FCC, "Spectrum policy task force report," Report of Federal Communications Commission, Et docket No. 02-135, November 2002.

[2] J. Mitola, "Cognitive radio: An integrated agent architecture for software defined radio," Ph.D. Thesis, Royal Institute of Technology, Sweden, May 2000.

[3] I. Akyildiz, W. Lee, M. Vuran, and M. Shantidev, "Next generation/ dynamic spectrum access/ cognitive radio wireless networks: a survey," Computer Networks (Elsevier) Journal, vol. 50, no. 4, pp. 2127-2159, September 2006.

[4] D. Chen, S. Yin, Q. Zhang, M. Liu, and S. Li, "Mining spectrum usage data: a large-scale spectrum measurement study," in Proc. of international conference on Mobile computing and networking, ACM Mobicom, 2009, Beijing, China, September 2009.
[5] S. M. Mishra, D. Cabric, C. Chang, D. Willkomm, B. V. Schewick A. Wolisz, and R. W. Brodersen, "A real time cognitive radio testbed for physical and link layer experiments," in Proc. of IEEE International Symposium on New Frontiers in Dynamic Spectrum Access Networks, DySPAN 2005, Baltimore, MD, November 2005.

[6] M. McHenry, "Spectrum white space measurements," New America Foundation Broadband Forum, June 20, 2003.

[7] M. A. McHenry, P. A. Tenhula, D. McCloskey, D. A. Roberson, and C. S. Hood, "Chicago spectrum occupancy measurements and analysis and a long-term studies proposal," in Proc. of TAPAS 2006, Boston, MA, August 2006.

[8] H. So, A. Tkachenko, and R. W. Brodersen, "A unified hardware/software runtime environment for fpga based reconfigurable computers using borph," in Proc. of International Conference on Hardware Software Codesign and System Synthesis, Seoul, Korea, October 2006.

[9] J. H. Reed, Software Radio: A Modern Approach to Radio Engineering. New York: Prentice Hall, May 2002.

[10] L. Duan, J. Huang, and B. Shou, "Cognitive mobile virtual network operator: Investment and pricing with supply uncertainty," in Proc. of IEEE Conference on Computer Communications, INFOCOM 2010, San Diego, CA, March 2010.

[11] Y. T. Hou, Y. Shi, and H. D. Sherali, "Optimal spectrum sharing for multi-hop software defined radio networks," in Proc. of IEEE Conference on Computer Communications, INFOCOM 2007, Anchorage, AL, May 2007.

[12] - , "Spectrum sharing for multi-hop networking with cognitive radios," IEEE Journal on Selected Areas in Communications, vol. 26, no. 1, pp. 146-155, January 2008.

[13] X. Liu and W. Wang, "On the characteristics of spectrum-agile communication networks," in Proc. of IEEE International Symposium on New Frontiers in Dynamic Spectrum Access Networks, DySPAN 2005, Baltimore, MD, November 2005.

[14] M. Alicherry, R. Bhatia, and L. E. Li, "Joint channel assignment and routing for throughput optimization in multi-radio wireless mesh networks," in Proc. of international conference on Mobile computing and networking, ACM Mobicom 2005, Cologne, Germany, August 2005.

[15] R. Draves, J. Padhye, and B. Zill, "Routing in multi-radio, multi-hop wireless mesh networks," in Proc. of international conference on Mobile computing and networking, ACM Mobicom 2004, Philadelphia, PA, September 2004.

[16] M. Kodialam and T. Nandagopal, "Characterizing the capacity region in multi-radio multi-channel wireless mesh networks," in Proc. of international conference on Mobile computing and networking, ACM Mobicom 2005, Cologne, Germany, August 2005.

[17] P. Kyasanur and N. H. Vaidya, "Capacity of multi-channel wireless networks: impact of number of channels and interfaces," in Proc. of international conference on Mobile computing and networking, ACM Mobicom 2005, Cologne, Germany, August 2005.

[18] X. Lin and N. B. Shroff, "Joint rate control and scheduling in multihop wireless networks," in Proc. of IEEE Conference on Decision and Control, Paradise Island, Bahamas, December 2004.

[19] — , "The impact of imperfect scheduling on cross-layer congestion control in wireless networks," IEEE/ACM Transactions on Networking, vol. 14, no. 2, pp. 302-315, April 2006.

[20] Z. Feng and Y. Yang, "Joint transport, routing and spectrum sharing optimization for wireless networks with frequency-agile radios," in Proc. of IEEE Conference on Computer Communications, INFOCOM 2009, Rio de Janeiro, Brazil, April 2009.

[21] M. R. Garey and D. S. Johnson, Computers and Intractability: A Guide to the Theory of NP-Completeness. New York, NY: W. H. Freeman and Company, 1979.

[22] M. Pan, Y. Song, P. Li, and Y. Fang, "Reward and risk for opportunistic spectrum accessing in cognitive radio networks," in Proc. of IEEE Global telecommunications conference, Globecom 2010, Miami, FL, December 2010.

[23] Y. Song, C. Zhang, and Y. Fang, "Stochastic traffic engineering in multihop cognitive wireless mesh networks," IEEE Transactions on Mobile Computing, vol. 9, no. 3, pp. 305-316, March 2010.

[24] A. Goldsmith, Wireless Communications. Cambridge, NY: Cambridge University Press, 2005.

[25] Y. Shi and Y.T. Hou, "A distributed optimization algorithm for multi-hop cognitive radio networks," in Proc. of IEEE Conference on Computer Communications, INFOCOM 2008, Phoenix, AZ, April 2008

[26] A. Papoulis and S. U. Pillai, Probability, Random Variables, and Stochastic Processes. McGraw-Hill, New York, 2002.

[27] G. Holton, Value-at-Risk: Theory and Practice. Academic Press, 2003.

[28] A. V. Oppenheim and R. W. Schafer, Discrete-Time Signal Processing, 3rd ed. New York: Prentice Hall, 2009. 